\title{
Treatment of chronic viral infection: tackling the problem from a new angle
}

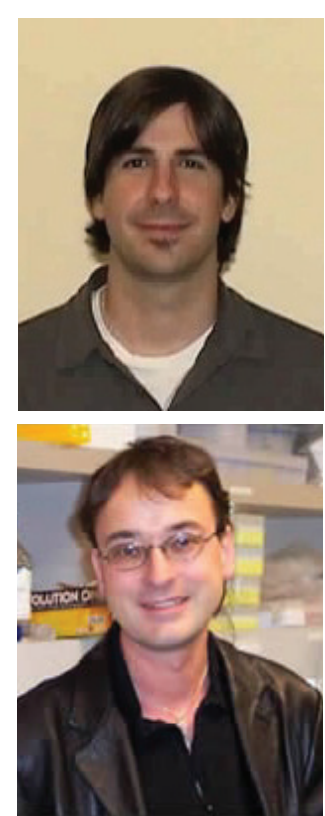

Christophe M Filippi \& Matthias $G$ von Herrath ${ }^{\dagger}$

${ }^{\dagger}$ Author for correspondence La Jolla Institute for Allergy \& Immunology,

9420 Athena Circle, La Jolla, CA 92037,USA

Tel.: +18587526817

Fax: +18587526993 matthias@liai.org '...complete elimination of the pathogen has remained elusive, despite the fact that antiviral immunity can be significantly increased in many instances.'

While humans have developed an immune system specialized in fighting microbial infections, certain pathogens can dramatically impair efficacy. This is notably the case for viruses such as HIV, hepatitis $\mathrm{C}$ virus $(\mathrm{HCV})$ or herpesviruses, which have evolved efficient immune escape properties. The resulting chronic infections can pose severe health problems. For example, chronic HCV infection causes liver damage and can lead to liver cancer. Reactivation of latent herpesviruses such as cytomegalovirus (CMV) or Epstein-Barr virus (EBV) can cause hepatitis, pneumonitis or even cancer. Finally, HIV infection invariably leads to the loss of immune function, thereby impairing the capacity of infected individuals to fight other infections (such as CMV). Numerous efforts have been undertaken to increase immunity in chronic viral infections with the goal of eliminating the pathogen, or at least reducing the immune-pathological consequences of viral persistence. Such vaccination strategies have been combined with direct antiviral drug treatments, such as protease inhibitors and highly active antiretroviral therapy in HIV infection, and interferon administration or ribavirin in $\mathrm{HCV}$ infection. In most of the situations where antiviral drugs are employed, viral loads can be significantly reduced and, for HIV, long-term deleterious consequences of the persistent infection are decreased. However, the outcome of ribavirin administration in chronic $\mathrm{HCV}$ is not quite as promising, since, although viral titers are lowered, liver fibrosis is enhanced, possibly as a direct result of the drug. Furthermore, and most importantly, complete elimination of the pathogen has remained elusive, despite the fact that antiviral immunity can be significantly increased in many instances. Where should we go from here? It appears that we need to consider revising our strategy somewhat, without overlooking the importance of antiviral drug therapy and the strengthening of $T_{H} 1 / T_{C} 1$ immunity to the virus.

\section{An alternative therapeutic strategy to current vaccine therapy}

As an alternative or addition to using interferon or antiviral drugs that can efficiently lower viral titers, immunomodulatory strategies conventionally consist of enhancing the antiviral response by directly inducing or amplifying virus-specific $\mathrm{T}$ cells. Although this approach may be effective in some scenarios, it has mostly failed to affect the outcome of chronic viral infections. In order to avoid recognition by the immune system, viruses use various strategies such as the active induction of immune suppression leading to the loss of T-cell function. Impairment of T-cell immunity is a common feature of chronic infections, raising the possibility that mechanisms of immune suppression may be conserved from one virus to the next. In support of this possibility, various chronic infections are associated with the systemic production of the cytokine interleukin (IL)-10, which impairs the function of $\mathrm{T}$ cells and antigen-presenting cells by inhibiting proinflammatory cytokine production, costimulation, major histocompatibility complex class II expression and chemokine secretion. IL-10 production has been observed during infection with $\mathrm{HCV}$, hepatitis B virus (HBV), CMV, EBV and more controversially, HIV. In some cases, the viral genome itself can encode an IL-10 homolog (CMV and EBV). The recent observation by ourselves and others that mice chronically infected with lymphocytic choriomeningitis virus (LCMV) produce large amounts of IL-10, led to the development of a new intervention. In our study, infection was resolved in the majority of mice treated with a blocking antibody to the IL-10 receptor (IL-10R). These mice developed a normal antiviral immune response, gained weight and returned to a healthy state as a consequence of neutralization of the IL-10 signaling pathway. Further analysis indicated that persistence of LCMV clone 13 was linked to a decline in the number of dendritic cells (DCs) belonging to the $\mathrm{CD} 8 \alpha^{+}$subset. CD8 $\alpha^{-}$DCs efficiently primed IL-10 secretion via virus-specific $\mathrm{CD} 4^{+} \mathrm{T}$ cells, preventing viral clearance, and, therefore enabling viral persistence. Anti-IL-10R therapy aborted the capacity of $\mathrm{CD} 8 \alpha^{-}$DCs to induce 
IL-10-secreting ' $\mathrm{T}_{\mathrm{R}}$ 1-like' cells, thereby enhancing $T_{H} 1 / T_{C} 1$ immunity and resolving infection in chronically infected mice.

Whether IL-10 affects the outcome of infection, the extent of immune pathology, the occurrence of further complications or indeed whether it could be the actual cause of persistence is unclear. IL-10 could either downregulate proinflammatory responses in a general manner or, more specifically, inhibit the induction or expansion of antiviral CD8 ${ }^{+}$T-cell effectors. Furthermore, it has been suggested that IL-10 may directly decrease the viability of $\mathrm{CD} 8 \alpha^{+} \mathrm{DCs}$, which we found induce potent antiviral immunity but are deleted during chronic LCMV infection. Regardless, conventional immunotherapy of persistent viral infections has, to date, been unsuccessful and this work suggests that tackling the problem from a different angle may be a crucial step toward the successful treatment of chronic infections in humans.

'...infection was resolved in the majority

of mice treated with a blocking antibody to the IL-10 receptor.'

\section{Blocking IL-10 to resolve other chronic infections: the case of $\mathrm{HCV}$}

We believe that comparable therapy in humans should primarily target $\mathrm{HCV}$ infection. The WHO estimates that 180 million people, some $3 \%$ of the world's population, are infected with $\mathrm{HCV}$, the majority of whom are chronic carriers. Chronic infection in patients who do not respond to conventional antiviral or interferon therapy can lead to liver damage or liver cancer. $\mathrm{HCV}$ is responsible for $50-75 \%$ of all liver cancer cases and two-thirds of all liver transplants in the developed world. Current estimates in the USA are that 3.9 million Americans are chronically infected with HCV. Hepatitis C has been compared with a 'viral time bomb'. It thus appears crucial to develop new strategies to successfully treat patients who do not respond to conventional therapy. It is known that systemic IL-10 production can be increased in chronic $\mathrm{HCV}$ infection and is most dramatic compared

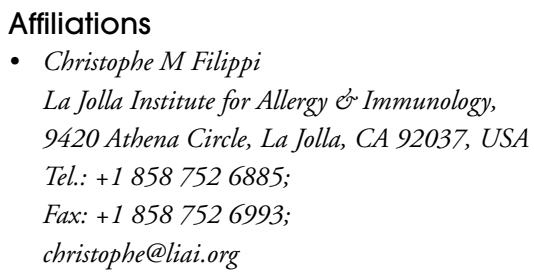

with other chronic viral infections. While patients who resolve $\mathrm{HCV}$ infection mount a robust antiviral $\mathrm{T}$-cell response, dysfunctional virus-specific effector $\mathrm{T}$-cell responses are observed in chronically-infected patients. Although the cause for antiviral T-cell dysfunction in virus persistence is not known, increased $\mathrm{HCV}$-specific IL-10 responses can be detected in patients with chronic hepatitis $\mathrm{C}$, and exogenous IL-10 therapy results in an increased $\mathrm{HCV}$ viral titer. Based on current knowledge, it should thus be determined whether anti-IL-10R blockade restores the function of antiviral $\mathrm{T}$ cells from HCV patients, first in vitro and then in clinical trials.

The next steps would be to combine viral vaccines, antiviral drugs or other antibodies to IL-10R blockade and determine whether synergy can be achieved in combating the viral disease with minimal side effects. It has been reported that program death (PD)-1 expression is associated with $\mathrm{HCV}$-specific $\mathrm{CD}^{+}{ }^{+} \mathrm{T}$-cell exhaustion in acute HCV infection. Furthermore, PD-1 receptor (PD-L1, B7-H1), which can mediate $\mathrm{T}$-cell apoptosis, is induced on hepatocytes by HCV infection. Similarly, exhausted antiviral $\mathrm{T}$ cells from mice chronically infected with LCMV express PD-1 and blockade of the PD-1/PD-1L signaling pathway results in resolution of the infection. We therefore propose that therapeutic agents that block the IL-10 signaling pathway and PD-1/PD-L1 interactions hold great promise for the treatment of persistent viral infections in humans, such as HCV and possibly HIV or CMV. This strategy may be particularly efficient when used in combination with conventional antiviral strategies, vaccine and/or other immunomodulatory agents. Such an innovative approach to treating persistent viral infections constitutes a departure from classical vaccine strategies that have unsuccessfully attempted to enhance antiviral immunity by enhancing $\mathrm{T}$-cell effectors directly. Importantly, targeting host factors that do not directly interact with the virus will limit the possibility that mutated resistant viral strains will emerge, as has been the case with a number of antiviral drugs.

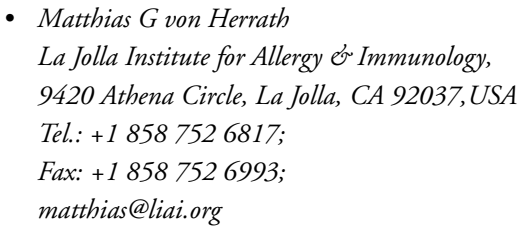

